\title{
Anticoagulación del circuito extracorpóreo esterilizado con óxido de etileno y rayos gamma: ¿necesitan las mismas dosis de heparina?
}

\author{
Sonia Muñoz Pilar - Marta Isabel San Juan Miguelsanz - Elena Conde Gómez - Verónica Gamarra González - Ángela García \\ Fuentes - Sergio Matesanz Sanchidrián
}

\section{Enfermeros/as}

Fundación Renal Iñigo Alvarez de Toledo, Centro de diálisis Los Olmos. Segovia

\section{Resumen}

Introducción: En los últimos años se han ido sustituyendo las líneas de hemodiálisis esterilizadas con óxido de etileno por las esterilizadas con rayos gamma, dado que numerosos autores han demostrado que es un método más biocompatible. Al utilizar líneas esterilizadas con rayos gamma observamos que se formaban más coágulos que con las esterilizadas con óxido de etileno.

Objetivos: Observar si hay diferencia en las dosis de heparina utilizadas y en la formación de coágulos en los circuitos extracorpóreos esterilizados con rayos gamma y óxido de etileno.

Material y método: Hemos realizado un estudio cuasi-experimental, longitudinal y prospectivo, utilizando en el mismo paciente líneas de hemodiálisis esterilizadas con rayos gamma y con óxido de etileno. Se realizaron en cada paciente 12 sesiones de hemodiálisis consecutivas con cada tipo de línea. Después de cada sesión se valoraron las pérdidas hemáticas en las cámaras arteriales y venosas, así como las necesidades de heparina.

\footnotetext{
Dirección para la correspondencia:

Sonia Muñoz Pilar

Calle Doctor Velasco No 27

40003 Segovia

e-mail: msanjuanmiguelsanz@yahoo.es
}

Resultado: Los resultados obtenidos demuestran que la formación de coágulos es mayor en las líneas esterilizadas con rayos gamma y que sí se modificó la dosis de heparina aunque no fue estadísticamente significativa.

Discusión: Mantener una buena anticoagulación del circuito extracorpóreo durante la diálisis permite: realizar un tratamiento clínicamente satisfactorio durante el tiempo requerido, evitar pérdidas estimadas altas y minimizar la heparina circulante disminuyendo los efectos secundarios.

PALABRAS CLAVE:

- LÍNEAS DE HEMODIÁLISIS

- ANTICOAGULACIÓN

- HEPARINA

- ÓXIDO DE ETILENO

- RAYOS GAMMA

- BIOCOMPATIBILIDAD 
Anticoagulation of the extracorporeal circuit sterilized with ethylene oxide and gamma irradiation: is the same dose of heparin needed?

\begin{abstract}
Introduction: In recent years, haemodialysis lines sterilized using ethylene oxide have been replaced with lines sterilized using gamma irradiation, as several authors have proven that it is a more biocompatible method. On using lines sterilized by gamma irradiation, we observed that more clots were formed than with lines sterilized with ethylene oxide.
\end{abstract}

Aims: To observe whether there is a difference in the heparin doses used and in the formation of clots in the extracorporeal circuits sterilized using gamma irradiation and ethylene oxide.

Material and method: We carried out a quasiexperimental, longitudinal and prospective study using haemodialysis lines sterilized by gamma irradiation and by ethylene oxide on the same patient. On each patient, 12 consecutive haemodialysis sessions with each type of line were carried out. After each session, the blood loss in the arterial and venous chambers was evaluated, together with the heparin needs.

Results: The results obtained show that clot formation is higher in the lines sterilized using gamma irradiation and that the heparin dose was indeed modified, although it was not statistically significant.

Discussion: Maintaining good anticoagulation of the extracorporeal circuit during dialysis makes it possible to: carry out a clinically satisfactory treatment during the time required, prevent estimated high losses and minimize the circulating heparin, reducing the side effects.

\section{KEY WORDS: \\ - HAEMODIALYSIS LINES \\ - ANTICOAGULATION \\ - HEPARIN \\ - ETHYLENE OXIDE \\ - GAMMA IRRADIATION \\ - BIOCOMPATIBILITY}

\section{Introducción}

En los últimos años las líneas de hemodiálisis esterilizadas con óxido de etileno (OE) se han ido sustituyendo por las esterilizadas con rayos gamma ( $R G$ ). Estudios más recientes han demostrado que el material médico-quirúrgico esterilizado con RG es más biocompatible que el esterilizado con $0 E^{1}$. En las técnicas de depuración extrarrenal, la sangre establece, durante prolongados períodos de tiempo, contacto con superficies no fisiológicas, lo que predispone a la activación de la coagulación ${ }^{2}$. En nuestra práctica diaria, hemos observado que en las cámaras de las líneas esterilizadas con $0 \mathrm{E}$ se formaban menos coágulos que en las esterilizadas con RG, con la consiguiente disminución de la dosis de heparina necesaria para mantener anticoagulado el circuito extracorpóreo.

La esterilización consiste en la reducción del número de microorganismos vivos presentes en cualquier medio a niveles tales que no sean capaces de manifestarse. Los procesos de esterilización convencionales comprenden calor seco, calor húmedo, irradiación o exposición al oxido de etileno $u$ otro agente ${ }^{3}$. Los dializadores y las líneas utilizadas en hemodiálisis (HD) se esterilizan mediante óxido de etileno, radiaciones gamma o vapor. Los agentes esterilizantes y los métodos de esterilización difieren ampliamente en su biocompatibilidad ${ }^{4}$.

La biocompatibilidad se puede definir como la capacidad de los materiales, instrumentos o sistemas, de actuar sin producir una alteración clínica significativa en el huésped. En la HD el término biocompatibilidad engloba toda una serie de interacciones específicas e inespecíficas que ocurren entre el enfermo y el procedimiento de diálisis ${ }^{5}$. Los fenómenos adversos actualmente aceptados como dependientes del grado de biocompatibilidad, se relacionan fundamentalmente con la activación del sistema del complemento y la generación de interleuquinas, aunque también con la actuación sobre distintas células sanguíneas (neutrófilos, monocitos, linfocitos T) y sobre la coagulación (fibrinólisis y activación plaquetaria) $^{6}$.

Durante el proceso de HD la sangre es conducida a través de un circuito extracorpóreo que lleva la sangre del paciente al dializador (línea arterial) y de nuevo hacia el paciente (línea venosa). Todos los circuitos extracorpóreos, incluidos los más biocompatibles, tienden a estimular y producir la coagulación de la 
sangre en su interior, por lo que la realización de la HD requiere el uso de anticoagulantes ${ }^{7}$. Además, evitando la formación de trombos, se garantiza que durante el proceso se mantengan los aclaramientos óptimos y se minimice la pérdida hemática. La heparina no fraccionada es el anticoagulante más utilizado para la hemodiálisis y se ha asociado con complicaciones como la trombocitopenia inducida por heparina. Los pacientes con elevado riesgo hemorrágico pueden dializarse sin heparina evitando flujos sanguíneos lentos y utilizando dializadores biocompatibles ${ }^{8}$.

Los siguientes procesos aumentan el riesgo de trombosis en el paciente y en el circuito?:

- Aumento de los niveles de fibrinógeno.

- Reducción de la actividad de la proteína C anticoagulante.

- Tratamiento con eritropoyetina.

- Naturaleza física y química del material que compone el circuito extracorpóreo.

- Flujo sanguíneo bajo.

- Hematocrito elevado.

- Tasa de ultrafiltración elevada.

- Trasfusión sanguínea intradiálisis.

- Infusión de lípidos intradiálisis.

Las consecuencias de la trombosis del circuito extracorpóreo pueden ser:

- Aumento de las pérdidas hemáticas del paciente, que puede dar lugar al agravamiento de la anemia.

- Desprendimiento de trombos.

- Pérdida de eficacia de diálisis.

- Cambio de algún elemento del circuito extracorpóreo durante la sesión (estrés para el paciente, aumento de la carga para el personal, aumento de los costes).

- Aumento de las necesidades de heparina y posibles efectos secundarios de esta.

Revisada la bibliografía encontramos estudios que relacionan los distintos métodos de esterilización con las diferentes membranas de hemodiálisis (dializadores), con la biocompatibilidad, y con los factores que inducen

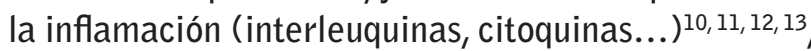
así como estudios que relacionan las necesidades de heparina con las diferentes membranas de $\mathrm{HD}^{14}$.

Macroscópicamente observamos que quedaban más coágulos en las cámaras de las líneas esterilizadas con $\mathrm{RG}$ que las esterilizadas por $0 \mathrm{E}$, por lo que nos planteamos los siguientes objetivos:

\section{Objetivos}

1. Valorar la formación de coágulos en las líneas de hemodiálisis esterilizadas con rayos gamma y con óxido de etileno.

2. Valorar la dosis de heparina necesaria para evitar la coagulación del circuito extracorpóreo esterilizado tanto con rayos gamma como por óxido de etileno.

\section{Material y métodos}

Se incluyeron en el estudio 23 pacientes de los cuales 4 fueron excluidos por ingreso hospitalario y 1 por trasplante renal. De los 18 pacientes que completaron el estudio 8 fueron hombres (44.4\%) y 10 mujeres (55.5\%), con una edad media de 70.8 años (rango: 44-87). La etiología de la insuficiencia renal crónica fue: Nefropatía intersticial 4 (22.2\%), glomerulonefritis crónica 4 (19\%), nefroangiosclerosis 4 (19\%), Nefropatía diabética 2 ( $11.1 \%)$, poliquistosis renal 2 $(9.5 \%)$, hereditaria $1(4.8 \%)$ y no filiada $1(4.8 \%)$. Entre las enfermedades asociadas 3 presentaban hepatopatía (14.\%) y un paciente presentaba enfermedad hematológica (4.8\%). El tiempo medio de estancia en programa de HD periódica fue de $53 \pm 59.2$ meses (rango: 4-233). El tiempo medio de duración de las sesiones de HD fue $236.42 \pm 14.9$ minutos (rango: 210-270). Los tipos de acceso vascular eran: 13 fístulas arterio-venosas autólogas, 1 prótesis de PTFE y 4 catéteres permanentes. Todos se dializaban con buffer de bicarbonato y la temperatura del baño de diálisis fue de $36^{\circ} \mathrm{C}$. Los pacientes se encontraban en tratamiento con eritropoyetina recombinante humana por vía intravenosa. La media del hematocrito fue $38.10 \pm 4.14$ para $R G$ y 37.84 para $0 E$ y de hemoglobina $12.38 \pm 1.39$ para $R G \mathrm{mg} / \mathrm{dl}$ y 12.20 para $0 \mathrm{E}$.

Diseño del estudio: Hemos realizado un estudio cuasiexperimental, longitudinal y prospectivo utilizando en el mismo paciente, líneas de hemodiálisis esterilizadas con diferentes métodos: esterilización con RG y esterilización con $0 \mathrm{E}$. Se registraron 36 sesiones de HD consecutivas: 12 con líneas esterilizadas con RG, 12 con líneas esterilizadas con $0 \mathrm{E}$ y las 12 últimas, de nuevo con líneas esterilizadas con RG. Después de cada sesión se valoraron y registraron las pérdidas hemáticas en las cámaras arteriales y venosas. La 
valoración se realizó por inspección visual según la siguiente clasificación: limpio (sin restos hemáticos), hilos (restos hemáticos adosados a las paredes de las cámaras), coágulos, tapón (coágulo en cámara venosa que impide el retorno de la sangre al paciente) y trombosis (sistema extracorpóreo coagulado). En las figuras 1,2 y 3 se muestran algunos ejemplos de cómo se han valorado las perdidas hemáticas. En función de la valoración realizada por cada enfermera se modificaban las dosis de heparina.

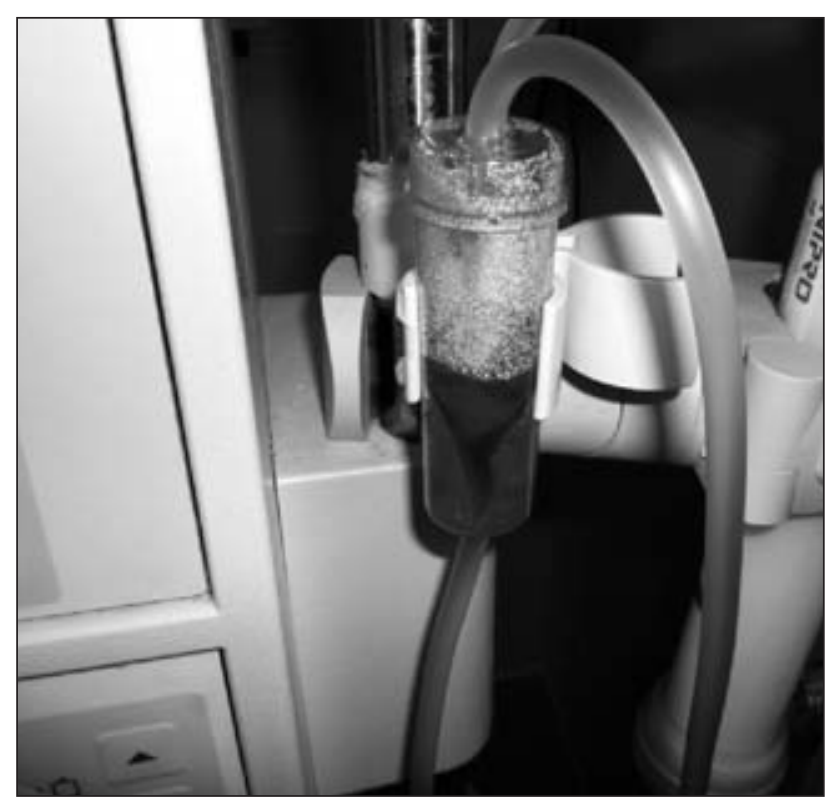

Figura 1: Cámara arterial con hilos (línea esterilizada con RG)

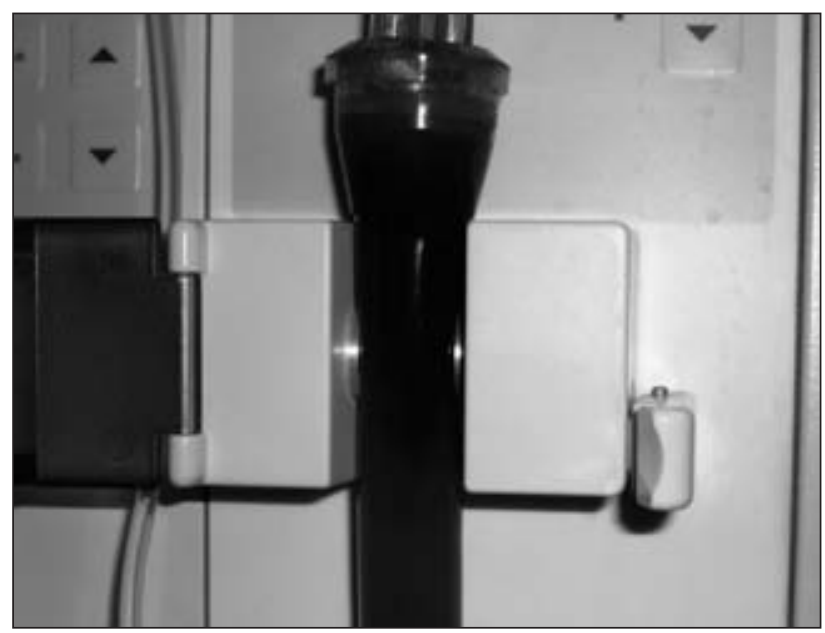

Figura 2: Cámara venosa con coágulo (línea esterilizada con RG)

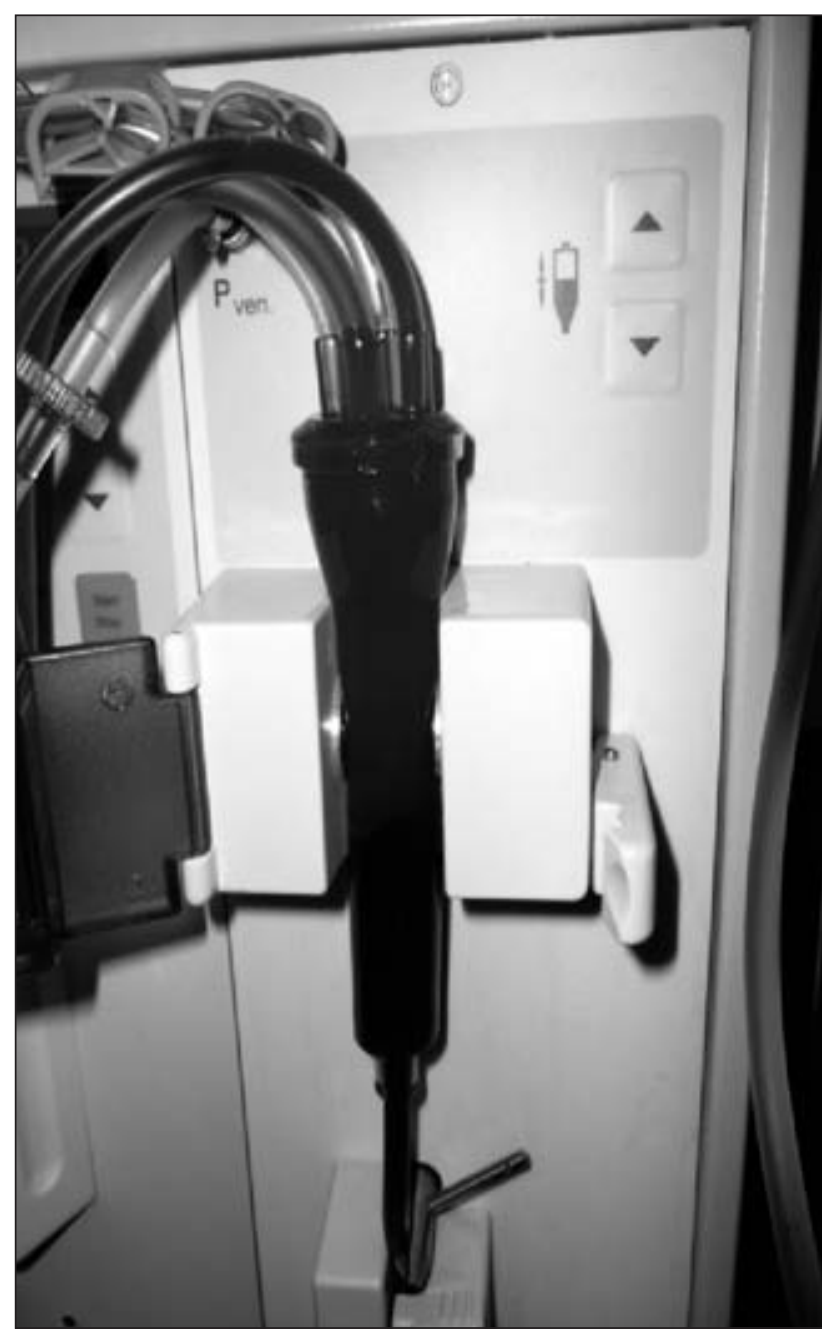

Figura 3: Cámara venosa con tapón (línea esterilizada con RG)

De los 18 pacientes, 3 no recibían ningún tipo de heparinización al encontrarse en tratamiento con acenocumarina (sintrom), 3 recibían una dosis heparina de bajo peso molecular al inicio de la HD y los doce pacientes restantes recibían bolos intermitentes de heparina sódica al $1 \%$.

Todos los pacientes utilizaban dializadores de polisulfona excepto un paciente alérgico a ella que usó un dializador de triacetato de celulosa. La preparación del circuito extracorpóreo se realizó mediante lavado con 1000 cc de suero fisiológico que contenía 5000 U/I de heparina. Durante el periodo de estudio la pauta del paciente no fue modificada en cuanto al tiempo, flujo de sangre que oscilo entre 300 y $400 \mathrm{ml} / \mathrm{min}$ y flujo de baño que oscilo entre 500 y $800 \mathrm{ml} / \mathrm{min}$ según la pauta médica del paciente. 
Al inicio de cada periodo del estudio, se realizo una analítica basal a cada paciente, incluyendo los siguientes parámetros: hemograma (hematocrito, hemoglobina y plaquetas), estudio de coagulación (actividad de Protrombina, tiempo de cefalina y fibrinógeno), PCR (proteína-C-reactiva para determinar estados inflamatorios) y adecuación de hemodiálisis (Kt/v y porcentaje de reducción de urea).

Análisis estadístico: Las variables categóricas se expresaron a través de porcentajes (\%) o frecuencias y se compararon con la prueba de chi-cuadrado. Las variables numéricas se describieron a través de la media, mediana, valores máximo y mínimos y desviación estándar.

La comparación de medias entre 2 grupos, se realizó a través de la prueba no paramétrica de rangos de Wilcoxon para muestras relacionadas o pareadas. La comparación de medias entre los 3 grupos, se realizó con la prueba no paramétrica de Friedman para muestras relacionadas o pareadas. Se consideró como significativo el valor de $p \leq 0.05$. Se utilizó el programa estadístico SPSS v. 15.0 para Windows.

\section{Resultados}

Los resultados obtenidos al comparar la cámara arterial y venosa de las líneas esterilizadas con RG y las esterilizadas con $0 \mathrm{E}$ se muestran en la tabla $\mathbf{l}$ y 2 respectivamente. La cámara arterial de las líneas esterilizadas con $0 \mathrm{E}$, presentaba menos restos hemáticos que las esterilizadas con RG.

Destacan considerablemente los resultados obtenidos con líneas esterilizadas con RG, en los que hay un

\begin{tabular}{|c|c|c|c|c|}
\hline \multirow[b]{2}{*}{ Camara Arterial } & \multicolumn{3}{|c|}{ Técnica de Esterilización* } & \multirow[b]{2}{*}{ Total } \\
\hline & $\begin{array}{c}\text { Rayos } \\
\text { Gamma } 1 \\
\text { n (\%) }\end{array}$ & $\begin{array}{c}\text { Oxido de } \\
\text { Etileno } \\
\text { n (\%) }\end{array}$ & $\begin{array}{c}\text { Rayos } \\
\text { Gamma } 2 \\
\text { n (\%) }\end{array}$ & \\
\hline Limpia & $10(32,3)$ & $17(54,8)$ & $4(14,9)$ & 31 \\
\hline Hilos de fibrina/coágulos & $6(30,0)$ & $1(5)$ & $13(65)$ & 20 \\
\hline Coágulos & $2(66,7)$ & $0(0)$ & $1(33,3)$ & 3 \\
\hline Total & 18 & 18 & 18 & 54 \\
\hline
\end{tabular}

Tabla 1. Distribución de los pacientes según Técnica de Esterilización y aspecto de la Cámara Arterial

\begin{tabular}{|c|c|c|c|c|}
\hline \multirow[b]{2}{*}{ Camara Arterial } & \multicolumn{3}{|c|}{ Técnica de Esterilización* } & \multirow[b]{2}{*}{ Total } \\
\hline & $\begin{array}{c}\text { Rayos } \\
\text { Gamma } 1 \\
\text { n(\%) }\end{array}$ & $\begin{array}{c}\text { Oxido de } \\
\text { Etileno } \\
\text { n (\%) }\end{array}$ & $\begin{array}{c}\text { Rayos } \\
\text { Gamma } 2 \\
\text { n (\%) }\end{array}$ & \\
\hline Limpia & $3(23,1)$ & $8(61,5)$ & $2(15,4)$ & 31 \\
\hline Hilos de fibrina/coágulos & $5(31,1)$ & $7(43,8)$ & $4(25,0)$ & 20 \\
\hline Coágulos & $10(40,0)$ & $3(12,0)$ & $12(48,0)$ & 3 \\
\hline Total & 18 & 18 & 18 & 54 \\
\hline
\end{tabular}

Tabla 2. Distribución de los pacientes según Técnica de Esterilización y aspecto de la Cámara Venosa 
elevado porcentaje de cámaras venosas que presentaron coágulos al final de la sesión de HD, frente a los resultados obtenidos para las cámaras venosas esterilizadas con $0 \mathrm{E}$, en las que la aparición de coágulos fue mucho menor.

Las dosis de heparina empleadas para prevenir la trombosis del circuito extracorpóreo, se bajaron durante el periodo de estudio con líneas esterilizadas con $0 \mathrm{E}$ y se subieron al utilizar de nuevo líneas esterilizadas con RG. Los resultados obtenidos de la valoración visual de la cámara arterial y venosa y de la dosis de heparina sódica $1 \%$ utilizada, se muestran en la tabla 3.

Los datos analíticos estudiados no se modificaron significativamente durante el periodo de estudio.

\section{Discusión}

La disminución de la necesidad de heparina y la ausencia de coagulación son características, entre otras, de la biocompatibilidad de una membrana de HD. Está descrito por numerosos autores ${ }^{11,15,16}$ que la esterilización con RG es mas biocompatible y segura que la de $0 \mathrm{E}$. Sin embargo, otros autores han estudiado la biocompatibilidad de las diferentes membranas de $\mathrm{HD}$ relacionándolo en algunas ocasiones con el tipo de material y con el proceso de esterilización. Álvarez Sadaba concluye que la HD afecta al endotelio y esto depende, en parte de la composición de la membrana y el esterilizante, señalando que la polisulfona esteri- lizada con $0 E$ es más biocompatible que la polisulfona esterilizada con vapor ${ }^{10}$.

Nuestro estudio demuestra que, en el proceso de la $H D$, las pérdidas hemáticas aumentan con el método que es más biocompatible ( $R G$ ), frente al que es menos biocompatible (OE). Sería importante destacar esta característica, sobre todo en los pacientes con accesos vasculares malfuncionantes o con riesgo de sangrado, en los que se emplean dosis mínimas de heparina o incluso sesiones de HD sin heparina. Por otro lado, aunque los resultados nos muestran que hemoconcentran más las líneas esterilizadas con $\mathrm{RG}$ que las esterilizadas con $0 \mathrm{E}$, hay que describir el diseño de las líneas esterilizadas con uno y otro método: en la cámara arterial de la línea esterilizada con RG, el segmento de línea que entra en la cámara se adosa a la pared, mientras que en la cámara arterial de la línea esterilizada con $0 \mathrm{E}$ se inserta en el centro de la cámara, sin estar adosada a la pared. Esto hace que, probablemente, no se formen ni hilos ni coágulos. No obstante, el diseño de las cámaras venosas, es igual en ambas líneas y los resultados muestran que hemoconcentran más los RG que el $0 \mathrm{E}$.

No podemos olvidar que existen otras razones que contribuyen a la aparición de coágulos en el circuito como son la modalidad de diálisis, el éxtasis sanguíneo, paradas de la bomba de sangre, hematocritos elevados, contacto de la sangre con el aire en las cámaras y un mal funcionamiento del acceso vascular.

\begin{tabular}{|c|c|c|c|c|c|}
\hline \multirow[b]{2}{*}{ Parámetros } & \multicolumn{3}{|c|}{ Técnica de Esterilización* } & \multicolumn{2}{|c|}{ Valor de $p^{*}$} \\
\hline & $\begin{array}{c}\text { Rayos } \\
\text { Gamma } 1 \\
\text { n (\%) }\end{array}$ & $\begin{array}{c}\text { Oxido de } \\
\text { Etileno } \\
\text { n (\%) }\end{array}$ & $\begin{array}{c}\text { Rayos } \\
\text { Gamma } 2 \\
\text { n (\%) }\end{array}$ & (1 vs 2) & (2 vs 3 ) \\
\hline Cámara Arterial & $1,56 \pm 0,7$ & $8(61,5)$ & $2(15,4)$ & 31 & \\
\hline Cámara Venosa & $5(31,1)$ & $7(43,8)$ & $4(25,0)$ & 20 & \\
\hline Dosis Heparina sódica** & $10(40,0)$ & $3(12,0)$ & $12(48,0)$ & 3 & \\
\hline
\end{tabular}

Tabla 3. Valoración visual de las cámaras arterial y venosa y de las dosis de Heparina sódica 1\% utilizadas según las técnicas de esterilización empleadas 
Michael Joannidis ${ }^{17}$ concluye que "la aparición prematura de coágulos en el circuito de hemodiálisis incrementa la pérdida de sangre, la carga de trabajo y los costes del tratamiento, por lo que mejorar y aumentar la supervivencia del circuito es clínicamente relevante". En nuestra opinión el estudio de aquellos factores que contribuyen a disminuir la trombosis del circuito extracorpóreo ayuda al personal de enfermería a realizar un tratamiento de hemodiálisis más seguro y con menos riesgos y evitaremos la aparición prematura de coágulos, que disminuye la eficacia de la HD, aumenta la anemia del paciente por las pérdidas sanguíneas ocasionadas y los requerimientos de eritropoyetina. Además si se llegara a producir la trombosis del circuito extracorpóreo, sería necesario preparar otra sesión de HD para ese paciente, incrementando la carga de trabajo y los costes de un tratamiento que, ya de por sí, es muy caro.

\section{Agradecimientos}

A todo el personal de enfermería del centro Los Olmos y en especial a la Dra. Astrid Rodríguez y al Dr. Jorge Rojas.

Recibido: Septiembre 2011

Revisado: Septiembre 2011

Modificado: Octubre 2011

Aceptado: Octubre 2011

\section{Bibliografía}

1. Martín Malo A. Biocompatibilidad de la diálisis. Llach y Valderrábano. Insuficiencia Renal Crónica. Diálisis y Trasplante Renal. Ediciones Norma. $2^{\mathrm{a}} \mathrm{Ed}$. Madrid 1997. Vol. 2. Cap. 34. Pág. 1049-1070.

2. Ronco C, Brendolan A, Lonneman G, Bellomo R, Piccinni $P$, Digito $A$ et all. Técnicas continúas en depuración extrarrenal en el tratamiento de la sepsis y otras causas de insuficiencia renal aguda. Jofré $R$, López J.M, Luño J, Pérez R, Rodríguez P. Tratado de hemodiálisis. $2^{a}$ Edición. Editorial Médica Jims. Barcelona 2006. Cap. 33. Pág. 678.

3. Díaz Alfonso, G.R. Estado actual de los procesos de esterilización. Memorias V Congreso de la Sociedad Cubana de Bioingeniería. Habana. 2003. Artículo T_0074.

4. Rodriguez-Benot A, Santamaría R, Martín-Malo A, Aljama P. Sterilization procedures and biocompatibility. Contrib Nephrol; (137): 138-45, 2002.

5. Valderrábano $\mathrm{F}$. El tratamiento de la Insuficiencia Renal Crónica en España. Nefrología. 1995. 14 suple 1:S27-35.

6. Varela Lema L, Ruano Raviña A. Efectividad y seguridad de las diferentes variantes de hemodiálisis y hemodiafiltración. Santiago de Compostela. Servicio Galego de Saúde, Axencia de Avaliación de Tecnoloxías Sanitarias de Galicia, avalia-t; 2005. Serie Avaliación de Tecnoloxías. Informe de avaliación: INF2005/03.

7. Botella García J. Manual de Nefrología Clínica. Editorial Massón S.A. Ed. $1^{a}$. Barcelona 2002. Cap.26. Pág. 259.

8. Keller F, Seemann J, Preusbof L, Offermann G. Risk factors of system clotting in heparin hemodialysis. Nephrol. Dial Transplant, 5: 802-807. 1990.

9. Daugirdas John T. Manual de Diálisis Editorial LWW Ed. 4a . Cap. 12. Pág. 203.

10. Alvarez Sádaba M.L, Valoración de la biocompatibilidad de diferentes membranas de hemodiálisis mediante el estudio de factores hemostáticos relacionados con la función endotelial en pacientes en hemodiálisis crónica. Facultad de medicina. Universidad de Navarra. 2002. http://www.cfnavarra,es/ salud/anales/textos/vol24/n2/resumen2.html.

11. Rodríguez-Benot A, Santamaría R, Martín-Malo A, Aljama P. Sterilization procedures and biocompatibility. Servicio de Nefrología, Hospital Universitario Reina Sofia, Córdova, Spain. Contrib Nephrol. 2002, (137): 138-45.

12. Aucella F, Vigilante M, Gatta G, Grandone E, Colaizzo D, Margaglione $M$ et all. Effects of ethylene oxide and steam sterilization on dialysis iduced cytokine release by cuprophan memebrane. Department 
of Nephrology and Dialysis. Italy. Artif Organs. 2002 Jun; 26(6):543-5.

13. Aucella F, Tetta C, Tessore V, De Nitti C, Vigilante M, Gatta $G$, et all. Is steam sterization really making any difference in dialysis iduced cytokine release? Department of Nephrology and Dialysis. Italy. Int $\mathrm{J}$ Artif Organs 2002 Sep;25(9):832-7.

14. Santos de $P, M{ }^{a} R$, Bravo Prieto $B$, Rodríguez San Bruno M, Muñoz Pilar S. Estudio de las necesidades de heparina con diferentes membranas de diálisis. FRIAT. Centro de Hemodiálisis Los Olmos. Segovia. Ponencia Congreso de la Sociedad Española de Enfermería Nefrológica. Sevilla. 1998.
15. Müller TF, Seitz M, Eckle I, lange H, Kolb G: Biocompatibiity differences with respect to the dialyzer sterilzation method. Nephron; 2: 139-142, 1998.

16. Baccheschi S, Sereni L, De Nitti C, Barbucci R, Tetta C. Blood tubing and cytokine production: effect of sterilization. Ren Fail. 2001 May-Jul; 23 (3-4): (411-8).

17. Michael Joannidis and Heleen M Oudemans-van Straaten. Clinical review: Patency of the circuit in continiuous renal replacement therapy. Clinical Care 2007: 218. 